\title{
A Novel Approach to Assessing Differentiation Degree and Lymph Node Metastasis of Extrahepatic Cholangiocarcinoma: Prediction Using a Radiomics-Based Particle Swarm Optimization and Support Vector Machine Model
}

Xiaopeng Yao ${ }^{1,2}$, PhD; Xinqiao Huang ${ }^{3}$, MD; Chunmei Yang ${ }^{3}, \mathrm{MD}$; Anbin Hu ${ }^{1,2}$, PhD; Guangjin Zhou ${ }^{4}$, BA; Mei $\mathrm{Ju}^{5}$, MA; Jianbo Lei ${ }^{1,6^{*}}, \mathrm{PhD}$; Jian Shu ${ }^{3^{*}}, \mathrm{PhD}$

\footnotetext{
${ }^{1}$ School of Medical Information and Engineering, Southwest Medical University, Luzhou, China

${ }^{2}$ Central Nervous System Drug Key Laboratory of Sichuan Province, Southwest Medical University, Luzhou, China

${ }^{3}$ Department of Radiology, The Affiliated Hospital of Southwest Medical University, Luzhou, China

${ }^{4}$ Department of Radiology, Peking University Third Hospital, Beijing, China

${ }^{5}$ School of Nursing, Southwest Medical University, Luzhou, Sichuan Province, China

${ }^{6}$ Center for Medical Informatics/Institute of Medical Technology, Peking University, Beijing, China

*these authors contributed equally
}

\section{Corresponding Author:}

Jian Shu, PhD

Department of Radiology

The Affiliated Hospital of Southwest Medical University

25 Taiping Street

Luzhou, 646000

China

Phone: 8618980253083

Email: shujiannc@163.com

\section{Related Article:}

This is a corrected version. See correction statement in: https://medinform.jmir.org/2021/1/e25337

\begin{abstract}
Background: Radiomics can improve the accuracy of traditional image diagnosis to evaluate extrahepatic cholangiocarcinoma (ECC); however, this is limited by variations across radiologists, subjective evaluation, and restricted data. A radiomics-based particle swarm optimization and support vector machine (PSO-SVM) model may provide a more accurate auxiliary diagnosis for assessing differentiation degree (DD) and lymph node metastasis (LNM) of ECC.

Objective: The objective of our study is to develop a PSO-SVM radiomics model for predicting DD and LNM of ECC.

Methods: For this retrospective study, the magnetic resonance imaging (MRI) data of 110 patients with ECC who were diagnosed from January 2011 to October 2019 were used to construct a radiomics prediction model. Radiomics features were extracted from T1-precontrast weighted imaging (T1WI), T2-weighted imaging (T2WI), and diffusion-weighted imaging (DWI) using MaZda software (version 4.6; Institute of Electronics, Technical University of Lodz). We performed dimension reduction to obtain 30 optimal features of each sequence, respectively. A PSO-SVM radiomics model was developed to predict DD and LNM of ECC by incorporating radiomics features and apparent diffusion coefficient (ADC) values. We randomly divided the 110 cases into a training group $(88 / 110,80 \%)$ and a testing group $(22 / 110,20 \%)$. The performance of the model was evaluated by analyzing the area under the receiver operating characteristic curve (AUC).

Results: A radiomics model based on PSO-SVM was developed by using 110 patients with ECC. This model produced average AUCs of 0.8905 and 0.8461 , respectively, for DD in the training and testing groups of patients with ECC. The average AUCs of the LNM in the training and testing groups of patients with ECC were 0.9036 and 0.8889 , respectively. For the 110 patients, this model has high predictive performance. The average accuracy values of the training group and testing group for DD of ECC were $82.6 \%$ and $80.9 \%$, respectively; the average accuracy values of the training group and testing group for LNM of ECC were $83.6 \%$ and $81.2 \%$, respectively.
\end{abstract}


Conclusions: The MRI-based PSO-SVM radiomics model might be useful for auxiliary clinical diagnosis and decision-making, which has a good potential for clinical application for DD and LNM of ECC.

(JMIR Med Inform 2020;8(10):e23578) doi: 10.2196/23578

\section{KEYWORDS}

PSO-SVM algorithm; magnetic resonance imaging; lymph node metastases; differentiation degree; extrahepatic cholangiocarcinoma; radiomics feature; algorithm; MRI; radiomics; lymph; cancer; oncology

\section{Introduction}

Cholangiocarcinoma is a highly aggressive neoplasm with a poor prognosis. Cholangiocarcinomas are commonly classified as either extrahepatic cholangiocarcinoma (ECC) or intrahepatic cholangiocarcinoma (ICC), on the basis of their anatomic position in regard to the second-order bile ducts. Generally, ECCs account for approximately $80-90 \%$ of diagnosed cases of cholangiocarcinoma [1]. Most (60\%-70\%) of ECCs are perihilar or "Klatskin" tumors, including the hepatic duct bifurcation; the rest of ECCs incorporate in the distal common bile duct [1].

Radical surgical resection is still the uniquely definitive and effective therapy for the long-term survival of patients with ECC. Patients with ECC show a low survival rate, attributed to hidden early clinical symptoms and a lack of effective nonsurgical therapeutic agents, which lead to local lymph vascular invasion and lymph node metastases (LNMs) [2]. In general, surgical resection with a cure expectation is associated with an 18\%-54\% 5-year survival rate for ECC [3-5]. Among clinicopathological features, tumor differentiation, positive lymph node, and lymphatic invasion were considered independent predictors of the overall survival rate of ECC [6-8]. Therefore, the accurate preoperative assessment of tumor pathological differentiation degree and lymph node status (especially lymph node status) could provide considerable help for the planning of treatment as soon as possible.

Ultrasonography, computerized tomography (CT), 18-fluorodeoxyglucose positron emission tomography/computerized tomography (18F-FDG-PET/CT), magnetic resonance imaging (MRI) and magnetic resonance cholangiopancreatography (MRCP), direct cholangiography, and endoscopy are traditional imaging methods for observing and diagnosing ECC [3,9]. MRI is regarded as a noninvasive and precise imaging modality for patients with ECC. MRI can provide information about lymph node metastases and survival results [10]. However, we should recognize some of the inherent defects of MRI. Traditional techniques mainly depend on radiologists' subjective visual and qualitative observations. Therefore, we still have no quantitative way of predicting pathological differentiation degree (DD) and LNM of ECC, including MRI [11]. More importantly, it's quite difficult to analyze the tremendous digital characteristics of the cells, physiology, and genetic variation of patients in the images, which cannot be distinguished by human eyes [12]. In current clinical studies, preoperative morphological features of lymph nodes, such as size, number, ratio, morphology, signal intensity, and lymph node changes, can be used to evaluate the preoperative lymph node status of ECC [13-15]. However, the accurate prediction method for assessing DD and LNM of ECC is incomprehensive.

By extracting traditional MRI, a large number of radiologic features can be obtained. Radiomics can be intuitively regarded as an approach that can quantify the conversion of visual image information into deep features $[16,17]$. This radiomics model is based on a machine-learning approach that can help doctors make the most accurate diagnosis by mining and analyzing radiological features. So far, radiomics have been successfully used to assist in decision making on the diagnosis and risk stratification of several types of cancer, such as hepatocellular carcinoma [18], glioma [19], rectal cancer [20], lung cancer [21], breast cancers [22], and thymic epithelial tumors [23]. Nonetheless, the diagnostic significance of radiomics in patients with ECC has not be evaluated.

In this paper, a radiomics model based on particle swarm optimization and a support vector machine (PSO-SVM) was developed for predicting DD and LNM of patients with ECC.

\section{Methods}

\section{Patient Selection}

We retrospectively collected a total of 110 consecutive patients' data (which included 60 men and 50 women) with ECC who underwent radical surgical resection between January 2011 and October 2019 at our hospital (The Affiliated Hospital of Southwest Medical University). Every inpatient underwent an abdominal MRI examination within 2 weeks before surgical resection, chemotherapy, or radiotherapy. With approval from the local Institutional Review Board and Ethics Committee, all features for patients with ECC were retrospectively investigated. Retrieved data included clinical symptoms, laboratory examination, surgery notes, MRI features, and pathological outcomes (including pathological DD and lymph node status). All identifying information in the records was deleted to protect patients' privacy.

The inclusion criteria were as follows: (1) All patients had pathologically confirmed ECC; (b) the regional LNMs dissection was performed during the operation; (3) abdominal MRI scans were obtained within 2 weeks before surgical resection, chemotherapy, or radiotherapy; and (4) the clinical and follow-up data were available. The final diagnosis of ECC was based on a combination of pathological examination results and MRI examination. Exclusion criteria were as follows: (1) the absence of preoperational MRI images; (2) obscure MRI images; (3) the presence of unidentified, inconspicuous lesions; (d) a lack of pathological DD or lymphatic status of ECC. 
Of the initial 172 patients with a pathological diagnosis of ECC from January 2011 to February 2019, we excluded 62 patients because of insufficient medical examination information, such as the absence of preoperational MRI images $(n=15)$, obscure MRI images $(n=24)$, the existence of unidentified, inconspicuous lesions $(n=5)$, and a lack of pathological DD or lymphatic status of ECC $(n=18)$. Consequently, 110 patients were used for DD and LNM of ECC. A flow diagram summarizing the study selection and inclusion is reported in Figure 1. The DD of ECC was divided into a high-risk differentiation group $(n=44)$ and a low-medium risk differentiation group $(n=66)$. The LNM of ECC was divided into a positive lymph node metastases group [LNM (+); $\mathrm{n}=79]$ and a negative lymph node metastases group [LNM (-); $n=31]$.

Figure 1. Flow diagram of patient cohort selection ( $\mathrm{n}=110)$. DD: differentiation degree; ECC: extrahepatic cholangiocarcinoma; LNM: lymph node metastases; MRI: magnetic resonance imaging.

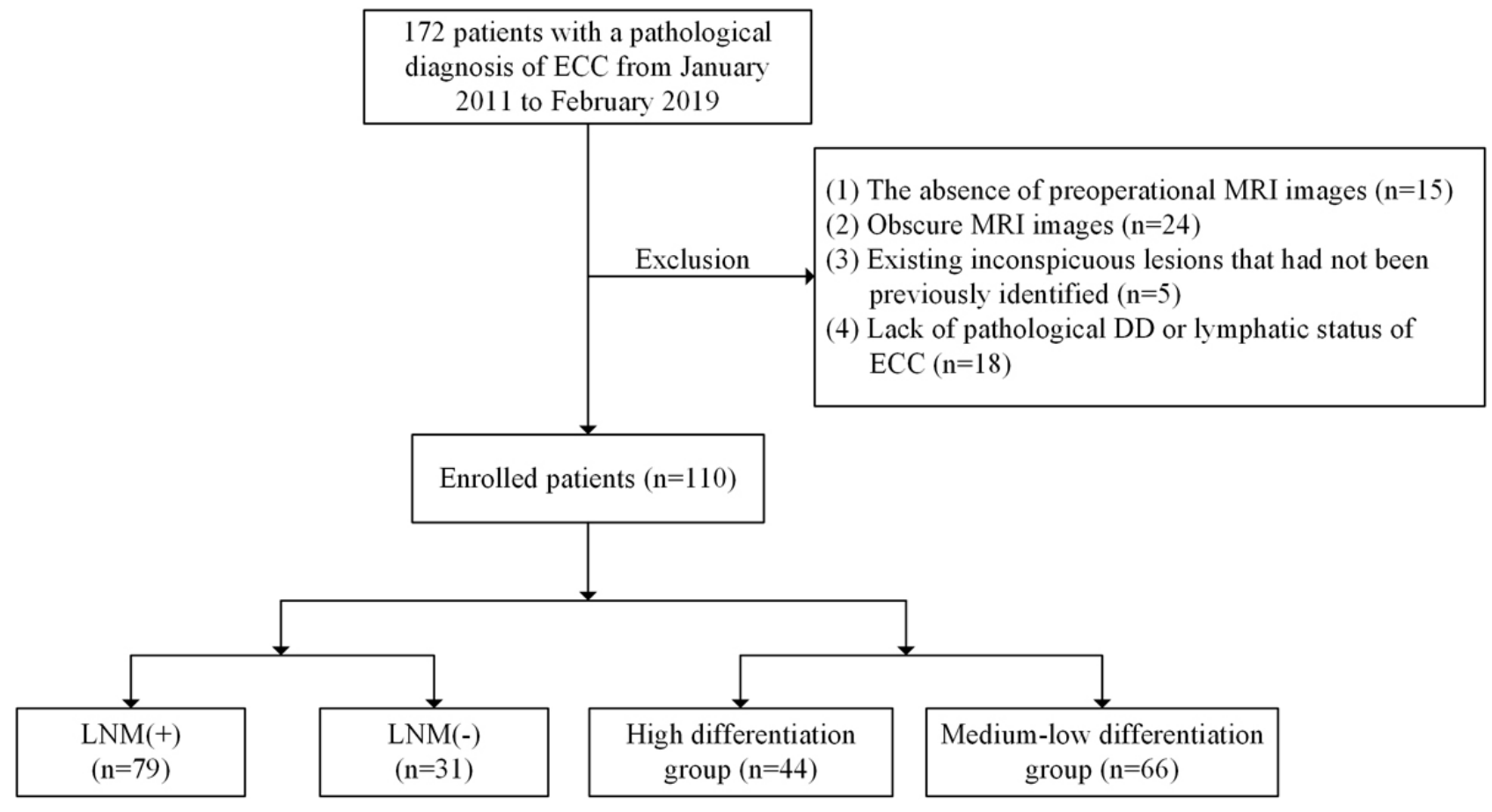

\section{Histopathologic Analysis of the Study Population}

All study patients underwent surgical resection, lesions were made into paraffin-embedded specimens, and the patients were histologically diagnosed with ECC. The samples were colored with a hematoxylin-eosin stain for regular histopathologic assessment. All specimens were identified by a seasoned histopathologist, who had over five years of work experience and was trained not to disclose individual participants' relevant information.

According to the American Joint Committee on Cancer (AJCC) and the College of American Pathologists, the ECC can be divided into 3 pathological grades: high-differentiation (G1), medium-differentiation (G2), and low-differentiation (G3) [24]. For G1, more than $95 \%$ of the tumor is composed of glands, and the perniciousness of the degree of the tumor is relatively low; for G2, 50-95\% of the tumor is composed of glands, and the degree of the tumor is moderately malignant; for G3, less than $50 \%$ of the tumor is composed of glands, and the perniciousness of the degree of the tumor is relatively large. This pathological differentiation has a certain significance for the clinical treatment and prognosis of ECC. Generally, G1 has a better prognosis and less metastasis than G2 and G3. G3 has a worse prognosis and more metastasis than G2.

\section{MRI Acquisition Protocol}

A Philips Achieva 3.0T superconducting MRI scanner with a quasar dual gradient system and a 16-channel phased-array torso coil was used to create all magnetic resonance images. Patients were asked to fast for 4-8 hours before the examination, with no restriction on drinking water. They also practiced breathing and holding their breath in the supine position. The imaging protocol mainly described the data acquisition and MRI sequences analysis. The MRI sequences were the following: an axial T1-weighted high-resolution isotropic volume excitation sequence (T1WI), an axial fat-suppressed turbo spin-echo (TSE) T2-weighted spectral attenuated inversion recovery (T2WI), a coronal TSE T2WI sequence, an axial dual-echo T1WI breath-hold gradient-echo sequence for the acquisition of in-phase and out-of-phase images, axial diffusion-weighted imaging (DWI), and T1-weighted dynamic contrast-enhanced MR images (including arterial, portal venous, transitional, and delayed phase). In this study, we mainly selected T1WI, T2WI, DWI, and ADC as the image data. The parameters of MRI sequences (T1WI, T2WI, DWI, ADC) are shown in Table 1. 
Table 1. The acquisition parameters of the abdominal magnetic resonance imaging (MRI) protocol.

\begin{tabular}{|c|c|c|c|c|}
\hline \multirow[t]{2}{*}{ Acquisition parameters } & \multicolumn{4}{|c|}{ Imaging protocol } \\
\hline & $\mathrm{T}_{1} \mathrm{WI}^{\mathrm{a}}$ & $\mathrm{T} 2 \mathrm{WI}^{\mathrm{b}}$ & $\mathrm{DWI}^{\mathrm{c}}$ & $\mathrm{ADC}^{\mathrm{d}}$ \\
\hline Repetition time (milliseconds) & 300 & 2610 & 2103 & $\mathrm{~N} / \mathrm{A}^{\mathrm{e}}$ \\
\hline Echo time (milliseconds) & 14 & 70 & 70 & N/A \\
\hline Flip angle (degrees) & 10 & 90 & 90 & N/A \\
\hline Field of view $(\mathrm{mm} \times \mathrm{mm})$ & $365 \times 305$ & $280 \times 305$ & $375 \times 305$ & N/A \\
\hline Matrix size $(\mathrm{mm} \times \mathrm{mm})$ & $204 \times 154$ & $176 \times 201$ & $128 \times 256$ & N/A \\
\hline Slice thickness $(\mathrm{mm}) / \mathrm{gap}(\mathrm{mm})$ & $7 / 1$ & $7 / 1$ & $7 / 1$ & N/A \\
\hline Slices (mm) & 24 & 24 & 72 & 24 \\
\hline Averaged number of signals & 1 & 2 & 4 & N/A \\
\hline$b$ values $\left(\mathrm{s} / \mathrm{mm}^{2}\right)$ & N/A & N/A & 0 and 800 & 800 \\
\hline
\end{tabular}

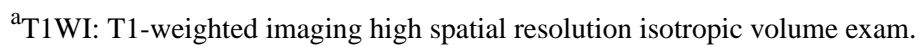

${ }^{\mathrm{b}}$ T2WI: fat-suppressed turbo spin-echo T2-weighted imaging spectral attenuated inversion recovery.

${ }^{\mathrm{c}}$ DWI: diffusion-weighted imaging.

${ }^{\mathrm{d}} \mathrm{ADC}$ : apparent diffusion coefficient.

${ }^{\mathrm{e}} \mathrm{N} / \mathrm{A}$ : not available.

\section{Workflow}

The workflow of this paper is shown in Figure 2. It includes five main parts: (1) imaging and region of interest (ROI) segmentation, (2) radiomics features extraction, (3) dimension reduction, (4) PSO-SVM model construct, and (5) data analysis. These 5 parts will be detailed in the following section.

Figure 2. Research workflow of the paper. ADC: apparent diffusion coefficient; DD: differentiation degree; DWI: diffusion-weighted imaging; ECC: extrahepatic cholangiocarcinoma; GLCM: grey-level co-occurrence matrix; LMN: lymph node metastases; PSO-SVM: particle swarm optimization and support vector machine; RLM: grey-level run-length matrix; ROI: receiver operating characteristic curve; T1WI: T1-weighted imaging high spatial resolution isotropic volume exam; T2WI: fat-suppressed turbo spin-echo T2-weighted imaging spectral attenuated inversion recovery.
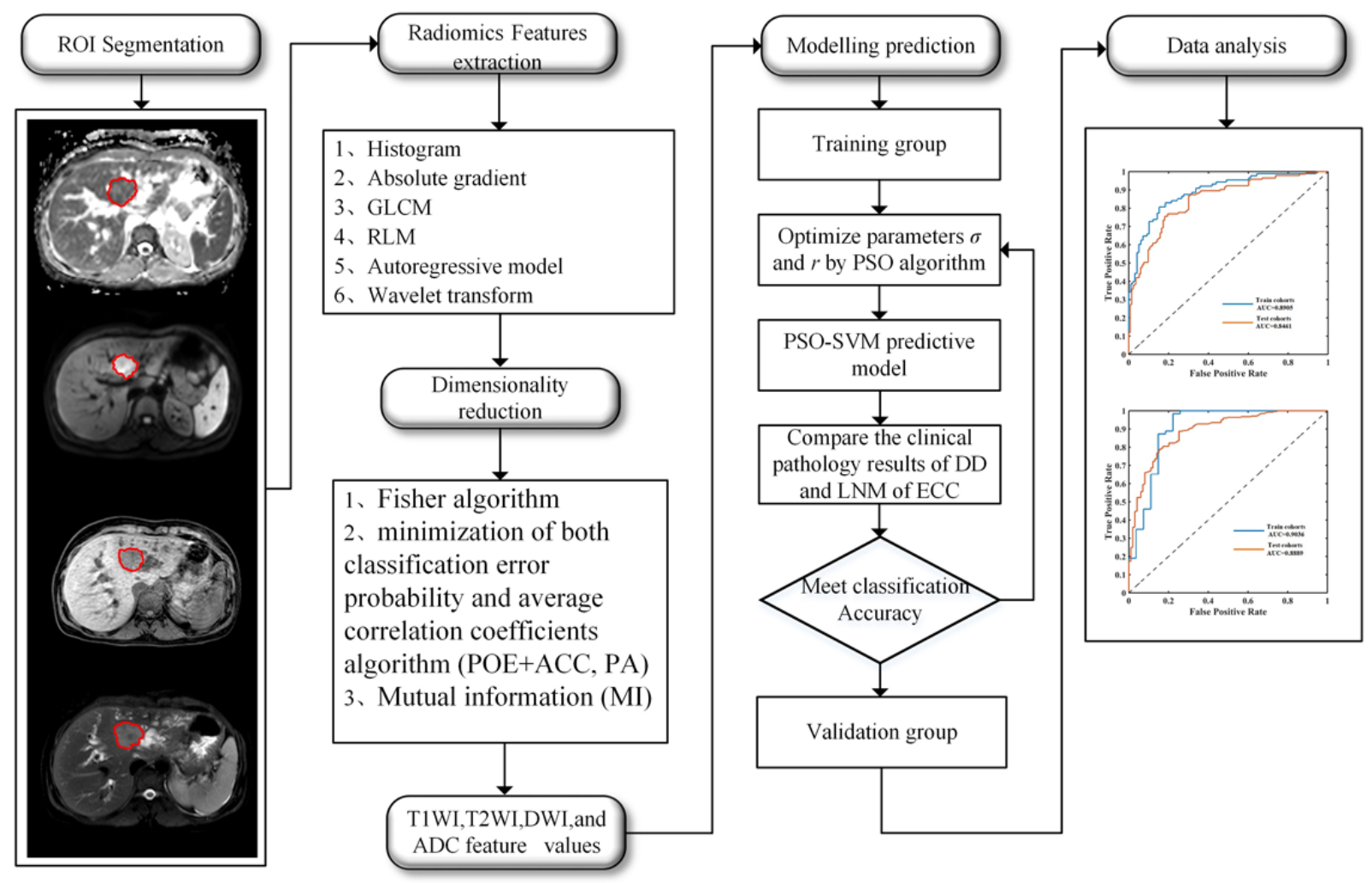


\section{ROI Segmentation}

All patients were followed up, and whether the lesion had recurred or metastasized was determined by radiological and pathological diagnosis. The relevant MRI images of patients were collected in the PACS-DICOM (picture archiving and communication system-Digital Imaging and Communications in Medicine) system, where the sequences of ECC were clearly selected. Given the 1515×1114-pixel image of cholangiocarcinoma, the average area of the lesions was 125.522 $\mathrm{mm}^{2}$. We did not exclude any images, and the radiology feature extraction used the entire ROI image.

\section{Radiomics Feature Extraction}

The MRI radiomics features of ECC were extracted using MaZda software (version 4.6; Institute of Electronics, Technical University of Lodz). The MRI analysis started with the definition of the ROIs. Under the guidance of an experienced radiologist, the ROI of the lesion was outlined to avoid adjacent vessels and bile ducts, and to locate the inside of the parenchyma of the tissue as much as possible. To outline lesions in MRI images, it is necessary to maintain about $1-2 \mathrm{~mm}$ from the edge of the tumor and to minimize the average volume of the surrounding structures when extracting image features. In the feature extraction process, the image intensity within the range of $\mu$ (SD 3) was normalized to minimize the influence of contrast and brightness variation. We finally extracted 300 radiomics features from the ROI of each sequence based on the following algorithms: first-order histogram, grey-level co-occurrence matrix (GLCM), grey-level run-length matrix (RLM), autoregressive model, and wavelet transform.

\section{Data Dimensionality Reduction}

All ROI features are high-dimensional data, and it may be difficult to select the required features if data dimensionality reduction (DDR) is not performed before the feature data is inputted into the classifier.

The purpose of DDR was to reduce the number of attributes under consideration so as to obtain the optimal features from the original features. Therefore, before we performed image classification and recognition, the significant features were selected to reduce the bias in features modeling. Based on MaZda software, we provided 3 methods for performing DDR and obtaining the optimal features: (1) the Fisher algorithm (F), (2) minimization of both classification error probability and the average correlation coefficients algorithm (POE+ACC, PA), and (3) mutual information (MI). These methods were used to deal with each feature separately and to remove almost indistinguishable features. Finally, 30 optimal features were selected from 300 radiomics features of each sequence (T1WI, T2WI, DWI, and ADC, respectively).

\section{PSO-SVM Model Construction}

After implementing DDR of the ROI features, the optimal features were adopted to build the prediction model. In the modeling process, all feature data had been normalized in the interval $(0,1)$ to eliminate the dimensional difference of radiomics features. The min-max normalization algorithm was used to normalize the radiomics features value cohort. In order to calculate uniformly, the main purpose was to convert the different magnitudes data into the same magnitude order. The min-max normalization algorithm can be described from the following equation:

$$
X=\left(x-x_{\min }\right) /\left(x_{\max }-x_{\min }\right)(1)
$$

$X$ is the normalized value of the optimal features, $x$ is the value of the optimal features, $x_{\max }$ is the maximum value of the optimal features, and $x_{\min }$ is the minimum value of the optimal features.

Because cholangiocarcinoma is a rare disease and the number of cases is relatively small, we faced a typical prediction modeling problem of small sample sizes. The basic principle of the PSO-SVM algorithm is to construct a hyperplane and distinguish high-dimensional mappings of feature data classification. The space of the feature data was taken as an input variable, and then the penalty parameters ( $\mathrm{c}$ and $\mathrm{g}$ ) of the support vector machine (SVM) were optimized by using the PSO algorithm. Then, the SVM algorithm was used to construct the prediction model for DD and LNM of ECC. To improve the performance of the prediction model, cross-validation and iterative training was used to verify data in this study.

\section{Data Analysis}

\section{Development, Performance, and Validation of a Radiomics Model}

In this paper, a radiomics model based on the PSO-SVM algorithm was established to predict DD and LNM of ECC by combining the optimal features of the tumor ROI and clinical outcomes. All patients were divided into high-risk and low-medium risk differentiated groups according to the pathological examination results. The min-max algorithm was used to normalize 120 features, including 90 radiomics features from 3 sequences (T1WI, T2WI, and DWI) and $30 \mathrm{ADC}$ values of the tumors, which can eliminate the negative effects caused by different sample dimensions. The distribution of DD and LNM cases of ECC was imbalanced. Statistically, there were mainly 2 methods to solve the problem: one was the under-sampling algorithm, and the other was the synthetic minority oversampling algorithm (SMOTE) [25]. The under-sampling algorithm could mainly achieve the sample balance by reducing the data set. This method was suitable for statistical problems with sufficient samples. Because there were fewer cases of ECC in this study, the under-sampling algorithm is not suitable for statistical problems with fewer samples. On the contrary, the oversampling algorithm was artificial to synthesize minority samples and add new samples to achieve sample balance. For the DD of ECC, the number of low-medium risk differentiated groups $(n=68)$ was significantly larger than that of high-risk differentiated groups $(n=42)$ for the DD of ECC, and the cases were extremely class-imbalanced. The number of low-medium-risk and high-risk differentiation groups were adjusted to be the same ( $n=1428)$ by using the SMOTE algorithm, respectively. For the LNM of ECC, the number of metastasis cases $(n=33)$ was significantly less than nonmetastasis cases $(n=77)$. Similarly, the numbers of metastasis and nonmetastatic groups were adjusted to be the same $(n=231)$ by using the SMOTE algorithm, respectively. In this way, the number of ECC cases was balanced. 
During the modeling process, we randomly selected 88 cases as the training group and the remaining 22 as the test group for DD and LNM of ECC. The PSO algorithm was used to obtain the optimal penalty parameters $\mathrm{c}$ of 7.3607 and $\mathrm{g}$ of 0.2132 so as to improve the classification accuracy and the robustness of this prediction model.

We determined the receiver operating characteristic curve (ROC) and the area under the curve (AUC) to evaluate the predictive performance of the PSO-SVM radiomics model. Furthermore, the sensitivity, specificity, positive predictive value (PPV), negative predictive value (NPV), and accuracy of the proposed model were calculated. Then, this model was evaluated by all of the above indicators for the validation cohort.

\section{Statistics, Comparison, and Analysis}

All continuous data (age and lesion area) were respectively given as means and medians (with interquartile ranges). ROC analysis was adopted to test the PSO-SVM model. We used the MATLAB statistics package (version 9.1; MathWorks) to conduct statistical analysis. We compared the result from the same case with independent $t$ tests and Wilcoxon rank sum tests, whereas the categorical variables, including gender and tumor location, were compared using a chi-square test. The evaluation indicators of the proposed model were also designed by
MATLAB, which included AUC, classification accuracy, PPV, NPV, sensitivity, and specificity. A 2-tailed $P$ value of less than .05 was considered statistically significant.

\section{Results}

\section{Clinical Features of the Studied Patients}

A total of 110 patients were selected from The Affiliated Hospital of Southwest Medical University. The mean age of patients was 57.0 (SD 10.0, range 28-83) years and the group included $60(54.5 \%)$ men and $50(45.5 \%)$ women. The clinical and baseline characteristics are summarized in Table 2. According to the pathological results of ECC, all patient cases were divided into high-risk differentiation groups $(n=42)$ and low-medium risk differentiation groups $(\mathrm{n}=68)$. Simultaneously, there were no significant heterogeneity differences between the 2 groups of data features for DD of ECC.

According to the pathological examination report, of the 110 patients, a total of 33 cases (30\%) were diagnosed with lymph node metastasis, and the other 77 cases $(70 \%)$ were diagnosed as being without lymph node metastasis. By analyzing the 5 characteristics in Table 2, there were no significant heterogeneity differences between the 2 groups of data features for non-LNM and LNM of ECC.

Table 2. Clinical and pathological characteristics of patients with extrahepatic cholangiocarcinoma (ECC; $n=110)$.

\begin{tabular}{|c|c|c|c|c|c|c|}
\hline \multirow[t]{2}{*}{ Characteristics } & \multicolumn{3}{|c|}{ Differentiation degree of ECC } & \multicolumn{3}{|c|}{$\mathrm{LNM}^{\mathrm{a}}$ of $\mathrm{ECC}$} \\
\hline & High-risk group & $\begin{array}{l}\text { Low-medium risk } \\
\text { group }\end{array}$ & $P$ value & Non-LNM & LNM & $P$ value \\
\hline Age in years, mean (SD) & $56.4(10.3)$ & $57.5(9.8)$ & .957 & $58.0(9.6)$ & $54.4(10.6)$ & .272 \\
\hline Gender, n (\%) & & & .434 & & & .969 \\
\hline Female & $22(50)$ & $28(42.4)$ & & $36(45.6)$ & $14(45.2)$ & \\
\hline Lesion location, n (\%) & & & .876 & & & .174 \\
\hline Porta & $20(45.5)$ & $29(43.9)$ & & $32(40.5)$ & $17(54.8)$ & \\
\hline Distal bile duct & $24(54.5)$ & $37(56.1)$ & & $47(59.5)$ & $14(45.2)$ & \\
\hline Lesion area ${ }^{b}\left(\mathrm{~mm}^{2}\right)$, mean $(\mathrm{SD})$ & $\begin{array}{l}115.144(\mathrm{SD} \\
78.425)\end{array}$ & $\begin{array}{l}131.8649(\mathrm{SD} \\
73.069)\end{array}$ & .495 & $\begin{array}{l}133.199(\mathrm{SD} \\
86.93)\end{array}$ & $\begin{array}{l}103.515(\mathrm{SD} \\
70.998)\end{array}$ & .816 \\
\hline
\end{tabular}

\footnotetext{
${ }^{\mathrm{a}}$ Lymph node metastases.

${ }^{\mathrm{b}}$ Lesion size was defined as the maximum diameter on transverse images.
}

\section{Reliability of Radiomics Feature Selection}

In order to construct a high-performance prediction model of PSO-SVM, we needed to obtain reliable ROI features. First, we randomly selected feature data of 30 patients from the 3 MRI sequences of T1WI, T2WI, and DWI, which had outlined ROI segmentation and extracted radiomics features. To evaluate the repeatability between intra-observer and inter-observer, we provided 2 radiologists (JS and $\mathrm{XH}$ ), each of whom have over 5 years of experience in abdominal oncologic imaging diagnosis. They performed ROI segmentation and feature extraction of the MRI images in a blinded fashion.
To ensure the objectivity of radiomics features, the 2 radiologists were aware of the diagnosis of ECC but were blinded to the clinical and pathologic details. The first radiologist repeatedly followed the same procedure to outline and determine the ROI twice within a week, and then we compared the 2 groups of radiomics features to evaluate intra-observer reliability. The second radiologist also independently outlined the ROI area and extracted radiomics features according to the same operating procedure. Then we evaluated inter-observer reliability by comparing the extracted results of the ROI area between the first radiologist and the second radiologist. The intraclass correlation coefficient (ICC) was used to evaluate the repeatability of radiomics features extracted by intra-observer 
and inter-observer. ICC can be obtained by using SPSS software according to the following equation:

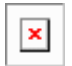

$\operatorname{Cov}(\mathrm{X}, \mathrm{Y})$ is covariance; $\sigma_{\mathrm{X}}$ is $\mathrm{X}$ standard deviation; $\sigma_{\mathrm{Y}}$ is $\mathrm{Y}$ standard deviation.

The radiomics features with ICC values of both the intra-observer and inter-observer greater than 0.75 (indicating satisfactory repeatability) were selected for subsequent modeling research. According to the above requirement, since all 300 radiation features extracted from each sequence have satisfactory consistency, no abnormal feature data were found and eliminated. The average value of the ICC within the inter-observer reached 0.97 (range $0.812-1, P<.001$ ), and the average ICC among the intra-observers reached 0.98 (range $0.826-1, P<.001$ ), as shown in Table 3 . According to the above calculation results, because the radiology features extracted in each sequence (T1WI, T2WI, DWI, ADC) have satisfactory consistency, no abnormal feature data was found and eliminated. Therefore, no abnormal characteristic data was found and eliminated.

Table 3. The intraclass correlation coefficient (ICC) between the intra-observer and inter-observer.

\begin{tabular}{lll}
\hline Data & Intra-observer & Inter-observer \\
\hline Patients, n & 30 & 30 \\
MRI sequence & T1WI, T2WI, DWI & T1WI, T2WI, DWI \\
ICC & & \\
$\quad$ Mean & 0.9849 & 0.9749 \\
$\quad$ Maximum & 0.9999 & 1 \\
$\quad$ Minimum & 0.8256 & 0.8641 \\
$\quad$ SD & 0.0278 & 0.0333 \\
\hline
\end{tabular}

\section{PSO-SVM Model Construction}

We selected 90 optimal features from 3 sequences (T1WI, T2WI, and DWI) and 30 ADC values by reducing dimensionality as the sample set. All of the data was normalized to be used for modeling. We randomly selected the optimal features of 88 patients as the training cohorts and the remaining optimal features of 22 patients as the test cohorts. The training cohorts were used to optimize the penalty parameters (c and g) of the SVM by using the PSO algorithm. To further improve the performance of the SVM classifier, the test cohorts were used to verify the performance and accuracy of the SVM classifier. Consequently, we built a radiomics prediction model based on PSO-SVM using the MRI images for predicting DD and LNM of ECC.

\section{Overall Validation of the PSO-SVM Radiomics Model}

In order to verify the robustness and deliverability of the PSO-SVM radiomics prediction model, we mainly evaluated the classification accuracy through the ROC curve. The ROC curve is a basic tool used for diagnostic test evaluation, which could reflect the performance of the PSO-SVM radiomics prediction model; it should ensure that the classification rates of the high-risk and low-medium-risk differentiated cases are as high as possible. However, the prediction model would make sure that a lot of the true positive cases are detected, even at the cost of some false positives during the screening phase.

Based on the PSO-SVM radiomics model, the performance of this model for predicting DD and LNM of ECC is shown in Figure 3, and the detailed data is listed in Table 4. The average accuracy of the training group and the testing group for DD of ECC were $82.6 \%$ and $80.9 \%$, respectively; the average sensitivity was $80.5 \%$ and $78.1 \%$, respectively; the average specificity was $83.1 \%$ and $81.5 \%$, respectively; the positive predictive value was $77.2 \%$ and $75.6 \%$, respectively; and the negative predictive value was $84.6 \%$ and $81.8 \%$, respectively. The average accuracy of the training group and the testing group for LNM of ECC was $83.6 \%$ and $81.2 \%$, respectively; the average sensitivity was $85.8 \%$ and $83.2 \%$, respectively; the average specificity was $82.1 \%$ and $79.6 \%$, respectively; the positive predictive value was $79.1 \%$ and $76.9 \%$, respectively; and the negative predictive value was $89.5 \%$ and $86.5 \%$, respectively. 
Figure 3. Receiver operating characteristic curves (ROC) of the performance evaluation for (a) differentiation degree prediction of extrahepatic cholangiocarcinoma in the training and testing cohorts and (b) lymphatic node metastasis of extrahepatic cholangiocarcinoma in the training and testing cohorts. AUC: area under the curve.

$\mathbf{a}$

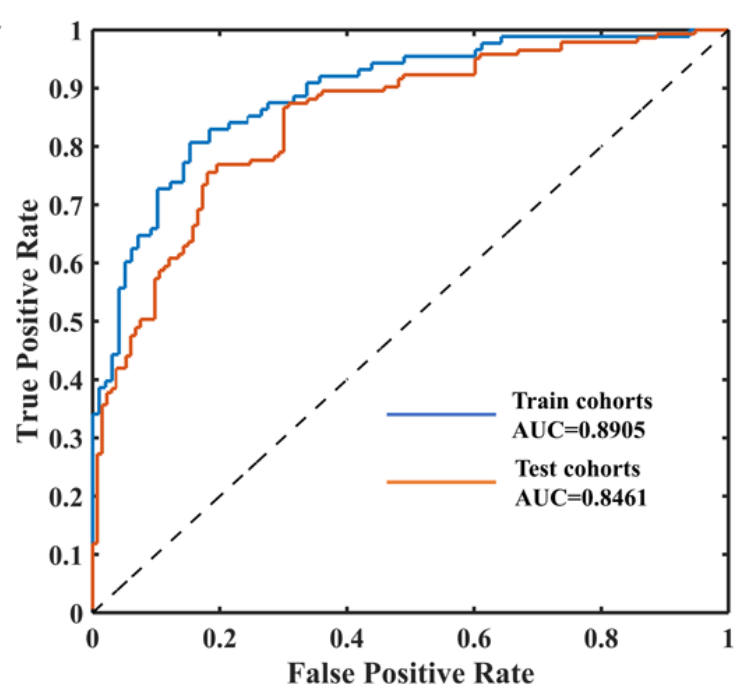

b

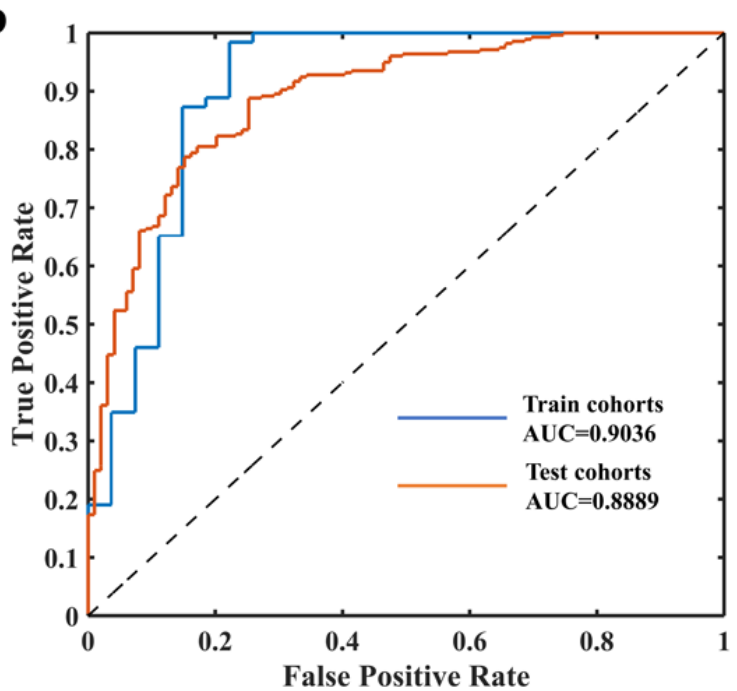

Table 4. The performance of the radiomics prediction model for predicting differentiation degree (DD) and lymph node metastases (LNM) of extrahepatic cholangiocarcinoma (ECC) by using a particle swarm optimization and support vector machine (PSO-SVM) model.

\begin{tabular}{|c|c|c|c|c|}
\hline \multirow[t]{2}{*}{ Evaluation indicators (\%) } & \multicolumn{2}{|l|}{ DD of ECC } & \multicolumn{2}{|l|}{ LNM of ECC } \\
\hline & Training group & Testing group & Training group & Testing group \\
\hline Average AUC ${ }^{\mathrm{a}}$ & $89.1^{\mathrm{b}}$ & 84.6 & $90.4^{\mathrm{b}}$ & 88.9 \\
\hline Average accuracy & 82.6 & 80.9 & 83.6 & 81.2 \\
\hline Average sensitivity ${ }^{\mathrm{c}}$ & 80.5 & 78.1 & 85.8 & 83.2 \\
\hline Average specificity ${ }^{\mathrm{d}}$ & 83.1 & 81.5 & 82.1 & 79.6 \\
\hline Average $\mathrm{PPV}^{\mathrm{e}}$ & 77.2 & 75.6 & 79.1 & 76.9 \\
\hline Average NPV ${ }^{\mathrm{f}}$ & 84.6 & 81.8 & 89.5 & 86.8 \\
\hline
\end{tabular}

${ }^{\mathrm{a}} \mathrm{AUC}$ : area under the curve.

${ }^{\mathrm{b}} P<.001$.

${ }^{\mathrm{c}}$ Sensitivity is computed at average radiologist specificity.

${ }^{\mathrm{d}}$ Specificity is computed at average radiologist sensitivity.

${ }^{\mathrm{e}} \mathrm{PPV}$ : positive predictive value; positive predictive value is computed at average radiologist sensitivity.

${ }^{\mathrm{f}} \mathrm{NPV}$ : negative predictive value.

\section{Discussion}

\section{Principal Findings}

We developed and validated a PSO-SVM prediction model for DD and LNM of ECC by using a radiomics approach. We performed this study to evaluate ECC and improved the efficiency of clinical diagnosis by using machine learning algorithms and a radiological approach. Our preliminary findings indicate that the radiological model incorporating the patients' MRI image sequence (T1WI, T2WI, DWI) and ADC values has superior diagnostic performance. The prediction performance of this model is shown in Figure 3. In the training and test groups, the average AUC of patients for high, medium, and low DD of ECC were 0.8905 and 0.8461 (the maximum AUC was 0.97), respectively. The average AUC of patients for LNM of ECC were 0.9036 and 0.8889 (with a maximum AUC of 1.00), respectively. Compared with the literature [20,26], our research results have higher prediction accuracy. The entire prediction model has the characteristics of multi-modality and high robustness, which comprehensively considered the radiomics feature of multiple sequences (T1WI, T2WI, DWI, 
ADC). Therefore, the proposed PSO-SVM prediction model can help clinicians choose an optimal treatment strategy, improve the prognosis of patients with ECC, and reduce complications, making it a potential postoperative evaluation tool in clinical practice.

It is generally recognized that imaging is the most important method for preoperative evaluation of ECC. However, traditional imaging methods have many defects in accurately evaluating the DD and LNM of ECC. The continuous development of ultrasonography, CT, 18-FDG PET/CT, and MRI technology in medical research have provided a great leap forward with respect to the LNM status of ECC [27-30]. Ercolani et al [29-31] reported that the sensitivity, specificity, and accuracy of CT examination of ECC were $35.2 \%, 91.8 \%$, and $46.1 \%$, respectively. Lewis et al $[32,33]$ showed that $\mathrm{CT}$ and MRI can evaluate the degree of pathological differentiation of ECC. However, the traditional techniques, which mainly rely on the subjective observation of radiologists, have many limitations. Transabdominal ultrasonography may only detect the dilatation of bile ducts in the majority of patients with intraductal tumors. CT can be used for X-ray imaging, but X-ray itself may be harmful to the health of patients. PET/CT is expensive and may be affected by false-positive results of benign lesions, such as biliary tract infection or sclerosing cholangitis [34,35]. Most importantly, it is difficult to analyze the tremendous digital characteristics of the biological features of patients in images using traditional techniques.

In contrast, radiomics can conquer these shortcomings. Researchers of radiomics can develop predictive models for clinical outcomes, such as survival, distant metastasis, and molecular feature classification [34-37], by mining potential associations between the quantitative features and pathophysiological characteristics of images [36-39]. According to our literature review, there is a sparse number of studies on DD and LNM that use a machine learning algorithm combined with radiomics to predict ECC, and the prediction accuracy is low. In this study, we innovatively proposed a PSO-SVM model based on radiomics to predict the DD and LNM of ECC. In the training and testing groups, the average prediction accuracy values of DD and LNM of patients with ECC were $82.6 \%$ and $83.6 \%$, respectively, and the average AUC values were 0.8680 and 0.89690 , respectively. The prediction results of this model were superior to those obtained from traditional image evaluation, such as ultrasonography, CT, 18-FDG PET/CT, and MRI technology. The results of our research indicate that the PSO-SVM model based on radiomics has potential clinical value as an auxiliary diagnostic method for the preoperative quantitative prediction of DD and LNM of ECC.

Furthermore, in order to use the extracted feature information to describe the shape and internal heterogeneity of the lesion area, the radiological features were integrated with the cellular and molecular features of the lesion to improve the accuracy of diagnosis prediction. So far, only a few studies have reported the relationship between the radiological features and the biological features of cholangiocarcinoma lesions. Researchers discovered that certain texture parameters correlate significantly with microvascular invasion, perineural invasion, differentiation, Ki-67, vascular endothelial growth factor, and cytokeratin 7 based on ultrasonography medical images [40]. They proposed radiomics signatures that have moderate efficiency in predicting the biological behaviors of cholangiocarcinoma noninvasively [40]. Gu-Wei Ji et al [41] regarded a radiomics model based on arterial phase CT scans as a valuable diagnostic tool to forecast LNM of ICC. Zhao et al [42] discovered that the combined model, containing enhancement MRI patterns, vascular endothelial growth factor (VEGFR), and radiomics features, showed a preferable early recurrence predictive performance compared to the radiomics model or clinic radiologic-pathological model alone, with AUC, sensitivity, and specificity values of $0.949,0.875$, and 0.774 , respectively. Liang et al [43] showed that the noninvasive radiomics nomogram developed using the radiomics signature and clinical stage could be used to predict early recurrence of ICC after partial hepatectomy. Compared with ultrasound and CT examination, MRI has become the imaging modality of choice for bile duct disease examination, especially for diagnosis and staging of cholangiocarcinoma. The contrast of high soft tissue helps to better discover and identify the infiltrating lesions. Magnetic resonance cholangiopancreatography (MRCP) is the most noninvasive method for evaluating bile ducts, allowing for assessments of tumor spread and the level of obstruction [44]. Dynamic contrast-enhanced MRI can not only provide crucial information about tumors, but it can also flag the appearance of distant metastasis and vascular invasion. The MRI examination can provide precise information on the biliary system, lesion range, and local tumor invasion.

As there were many differences between ECC and other liver lesions, such as origin, morbidity, growth pattern, imaging features, and tumor prognosis, the single evaluation method of ECC using radiological characteristics is prone to diagnostic blind spots. Since the ADC value could describe the diffusion capacity of water molecules in the lesion cells, the tissue structure and functional location of the lesion at the cellular and molecular level could be evaluated by combining the ADC value and radiological characteristics. Therefore, another innovation of this study is that we innovatively integrated 90 radiomics features from 3 MRI sequences (T1WI, T2WI, and DWI) and 30 ADC values to improve the prediction accuracy of the PSO-SVM model. At the same time, during the entire training process, the algorithm was repeatedly optimized with 200 iterations to ensure the reliability of the model. Therefore, our model can provide clinicians with auxiliary decision-making for ECC and provide a more personalized treatment plan for patients.

\section{Limitations}

The proposed research has certain limitations and deficiencies. First, since ECC is a rare disease, all patients were obtained from a single medical institution (The Affiliated Hospital of Southwest Medical University) for our study, and the sample number of the cases was relatively small. In order to further improve the accuracy and robustness of the prediction model, the next research work is mainly dedicated to collecting more patient data from other medical institutions. Secondly, the design of the study was retrospective in this paper; thus, there were missing data regarding clinical factors and disease progression. Finally, this model has certain predictive barriers in this study, 
which cannot make multi-modal prediction results for patients with time-variance. As the radiomics diagnosis is a systematic project, the models should take into account as many factors as possible, and the radiomics features should be correlated with other clinical results, such as biochemical examination, pathology, radiology, and genomic features, and provide quantitative clinical analysis results. With the development of various hospital information technologies and personal wearable devices, it has become more feasible to use real-time collected health data for comprehensive health management $[45,46]$ or hospital data to support intelligent auxiliary diagnosis and decision-making. Therefore, the multi-modal and big data prediction model for ECC will become the focus of the next research study.

\section{Conclusions}

In this paper, we developed a PSO-SVM radiomics model that incorporates the qualitative and quantitative radiomics features and pathological characteristics for predicting DD and LNM of ECC. The techniques used include image sketching, ROI region segmentation, feature extraction, dimension reduction, preprocessing, and classification. This model has the advantages of a simple principle, low computational cost, good robustness, and less manual intervention. The prediction result of the PSO-SVM radiomics model might be useful in the assistance of clinical diagnosis and decision-making, and the guidance of patients toward more individualized and accurate treatment.

\section{Acknowledgments}

This work was supported by Sichuan Science and Technology Program (Grant No. 2020YJ0151), the Health Committee of Sichuan province (Grant No. 19PJ151), the Applied Basic Research Program of Southwest Medical University (Grant No.2017-ZRZD-019), and the National Natural Science Foundation of China (NSFC) (Grants NO. 81771937 and No. 81871455).

We thank the staff of the Department of Radiology at the Affiliated Hospital of Southwest Medical University and the Center for Medical Informatics of Peking University for technical assistance.

This study was also partly supported by PKU-Baidu Fund project of Intelligent auxiliary diagnosis using medical images and Research project of constructing health big data platform and service system for medical and nursing combined elderly care institutions.

\section{Authors' Contributions}

JS and JL were guarantors of the integrity of the entire study. All the authors were involved in the formulation of the study concepts, study design, data acquisition, and data analysis and interpretation. All the authors were involved in manuscript drafting, manuscript revision, or approval of the final version of the manuscript. GZ, XH, CY, and XY participated in literature research. JS reviewed clinical studies. All authors were involved in experimental studies. XY performed statistical analysis and manuscript editing.

\section{Conflicts of Interest}

None declared.

\section{References}

1. Clements O, Eliahoo J, Kim JU, Taylor-Robinson SD, Khan SA. Risk factors for intrahepatic and extrahepatic cholangiocarcinoma: A systematic review and meta-analysis. Journal of Hepatology 2020 Jan;72(1):95-103. [doi: 10.1016/j.jhep.2019.09.007]

2. Lin H, Yang L, Tian F, Nie S, Zhou H, Liu J, et al. <p >Up-regulated LncRNA-ATB regulates the growth and metastasis of cholangiocarcinoma via miR-200c signals </p>. OTT 2019 Sep;Volume 12:7561-7571. [doi: 10.2147/ott.s217676]

3. Esnaola NF, Meyer JE, Karachristos A, Maranki JL, Camp ER, Denlinger CS. Evaluation and management of intrahepatic and extrahepatic cholangiocarcinoma. Cancer 2016 Jan 22;122(9):1349-1369. [doi: 10.1002/cncr.29692]

4. Andrianello S, Paiella S, Allegrini V, Ramera M, Pulvirenti A, Malleo G, et al. Pancreaticoduodenectomy for distal cholangiocarcinoma: surgical results, prognostic factors, and long-term follow-up. Langenbecks Arch Surg 2015 Jul 2;400(5):623-628. [doi: 10.1007/s00423-015-1320-0]

5. Kiriyama M, Ebata T, Aoba T, Kaneoka Y, Arai T, Shimizu Y, et al. Prognostic impact of lymph node metastasis in distal cholangiocarcinoma. Br J Surg 2015 Jan 22;102(4):399-406. [doi: 10.1002/bjs.9752]

6. Suzuki S, Shimoda M, Shimazaki J, Maruyama T, Oshiro Y, Nishida K, et al. $<\mathrm{p}>$ Number of positive lymph nodes and lymphatic invasion are significant prognostic factors after pancreaticoduodenectomy for distal cholangiocarcinoma $</ \mathrm{p}>$. CEG 2019 Jun;Volume 12:255-262. [doi: 10.2147/ceg.s207333]

7. $\mathrm{Hu}$ H, Jin Y, Shrestha A, Ma W, Wang J, Liu F, et al. Predictive factors of early recurrence after R0 resection of hilar cholangiocarcinoma: A single institution experience in China. Cancer Med 2019 Mar 13;8(4):1567-1575. [doi: 10.1002/cam4.2052]

8. Kato Y, Takahashi S, Gotohda N, Konishi M. Prognostic Impact of the Initial Postoperative CA19-9 Level in Patients with Extrahepatic Bile Duct Cancer. J Gastrointest Surg 2016 Jun 1;20(8):1435-1443. [doi: 10.1007/s11605-016-3180-5] 
9. Doherty B, Nambudiri VE, Palmer WC. Update on the Diagnosis and Treatment of Cholangiocarcinoma. Curr Gastroenterol Rep 2017 Jan 21;19(1). [doi: 10.1007/s11894-017-0542-4]

10. Jhaveri KS, Hosseini-Nik H. MRI of cholangiocarcinoma. J. Magn. Reson. Imaging 2014 Dec 01;42(5):1165-1179. [doi: 10.1002/jmri.24810]

11. Hosny A, Parmar C, Quackenbush J, Schwartz LH, Aerts HJWL. Artificial intelligence in radiology. Nat Rev Cancer 2018 Dec;18(8):500-510 [FREE Full text] [doi: 10.1038/s41568-018-0016-5] [Medline: 29777175]

12. Jiang F, Jiang Y, Zhi H, Dong Y, Li H, Ma S, et al. Artificial intelligence in healthcare: past, present and future. Stroke Vasc Neurol 2017 Dec;2(4):230-243 [FREE Full text] [doi: 10.1136/svn-2017-000101] [Medline: 29507784]

13. Nanashima A, Sakamoto I, Hayashi T, Tobinaga S, Araki M, Kunizaki M, et al. Preoperative Diagnosis of Lymph Node Metastasis in Biliary and Pancreatic Carcinomas: Evaluation of the Combination of Multi-detector CT and Serum CA19-9 Level. Dig Dis Sci 2010 Mar 18;55(12):3617-3626. [doi: 10.1007/s10620-010-1180-y]

14. Noji T, Kondo S, Hirano S, Tanaka E, Suzuki O, Shichinohe T. Computed tomography evaluation of regional lymph node metastases in patients with biliary cancer. Br J Surg 2007 Sep 13;95(1):92-96. [doi: 10.1002/bjs.5920]

15. Kiriyama M, Ebata T, Aoba T, Kaneoka Y, Arai T, Shimizu Y, et al. Prognostic impact of lymph node metastasis in distal cholangiocarcinoma. Br J Surg 2015 Jan 22;102(4):399-406. [doi: 10.1002/bjs.9752]

16. Sollini M, Antunovic L, Chiti A, Kirienko M. Towards clinical application of image mining: a systematic review on artificial intelligence and radiomics. Eur J Nucl Med Mol Imaging 2019 Jun 18;46(13):2656-2672. [doi: 10.1007/s00259-019-04372-x]

17. Gillies RJ, Kinahan PE, Hricak H. Radiomics: Images Are More than Pictures, They Are Data. Radiology 2016 Feb;278(2):563-577. [doi: 10.1148/radiol.2015151169]

18. Jiang H, Liu X, Chen J, Wei Y, Lee JM, Cao L, et al. Man or machine? Prospective comparison of the version 2018 EASL, LI-RADS criteria and a radiomics model to diagnose hepatocellular carcinoma. Cancer Imaging 2019 Dec 5;19(1). [doi: 10.1186/s40644-019-0266-9]

19. Li L, Mu W, Wang Y, Liu Z, Liu Z, Wang Y, et al. A Non-invasive Radiomic Method Using 18F-FDG PET Predicts Isocitrate Dehydrogenase Genotype and Prognosis in Patients With Glioma. Front. Oncol 2019 Nov 14;9. [doi: 10.3389/fonc.2019.01183]

20. Bibault J, Giraud P, Housset M, Durdux C, Taieb J, Berger A, et al. Author Correction: Deep Learning and Radiomics predict complete response after neo-adjuvant chemoradiation for locally advanced rectal cancer. Sci Rep 2018 Nov 12;8(1). [doi: 10.1038/s41598-018-35359-7]

21. Cong M, Feng H, Ren J, Xu Q, Cong L, Hou Z, et al. Development of a predictive radiomics model for lymph node metastases in pre-surgical CT-based stage IA non-small cell lung cancer. Lung Cancer 2020 Jan;139:73-79. [doi: 10.1016/j.lungcan.2019.11.003]

22. Li H, Zhu Y, Burnside ES, Huang E, Drukker K, Hoadley KA, et al. Quantitative MRI radiomics in the prediction of molecular classifications of breast cancer subtypes in the TCGA/TCIA data set. npj Breast Cancer 2016 May 11;2(1). [doi: 10.1038/npjbcancer.2016.12]

23. Xiao G, Rong W, Hu Y, Shi Z, Yang Y, Ren J, et al. MRI Radiomics Analysis for Predicting the Pathologic Classification and TNM Staging of Thymic Epithelial Tumors: A Pilot Study. American Journal of Roentgenology 2020 Feb;214(2):328-340. [doi: 10.2214/ajr.19.21696]

24. Edge SB, Compton CC. The American Joint Committee on Cancer: the 7th Edition of the AJCC Cancer Staging Manual and the Future of TNM. Ann Surg Oncol 2010 Feb 24;17(6):1471-1474. [doi: 10.1245/s10434-010-0985-4]

25. Elreedy D, Atiya AF. A Comprehensive Analysis of Synthetic Minority Oversampling Technique (SMOTE) for handling class imbalance. Information Sciences 2019 Dec;505:32-64. [doi: 10.1016/j.ins.2019.07.070]

26. Lewis S, Besa C, Wagner M, Jhaveri K, Kihira S, Zhu H, et al. Prediction of the histopathologic findings of intrahepatic cholangiocarcinoma: qualitative and quantitative assessment of diffusion-weighted imaging. Eur Radiol 2017 Dec 12;28(5):2047-2057. [doi: 10.1007/s00330-017-5156-6]

27. Meng Z, Lin X, Zhu J, Han S, Chen Y. A nomogram to predict lymph node metastasis before resection in intrahepatic cholangiocarcinoma. Journal of Surgical Research 2018 Jun;226:56-63. [doi: 10.1016/j.jss.2018.01.024]

28. Ercolani G, Grazi GL, Ravaioli M, Grigioni WF, Cescon M, Gardini A, et al. The Role of Lymphadenectomy for Liver Tumors. Annals of Surgery 2004;239(2):202-209. [doi: 10.1097/01.sla.0000109154.00020.e0]

29. Li X, Zhang Y, Zhang Y. 18F-FDG PET/CT may be a suitable method for preoperative diagnosis and evaluation of Chinese older patients with hilar cholangiocarcinoma. BMC Geriatr 2018 Jul 6;18(1). [doi: 10.1186/s12877-018-0846-8]

30. D’Antuono F, De Luca S, Mainenti PP, Mollica C, Camera L, Galizia G, et al. Comparison Between Multidetector CT and High-Field 3T MR Imaging in Diagnostic and Tumour Extension Evaluation of Patients with Cholangiocarcinoma. J Gastrointest Canc 2019 Jul 29;51(2):534-544. [doi: 10.1007/s12029-019-00276-z]

31. $\mathrm{Hu} \mathrm{H}$. Prognostic factors and long-term outcomes of hilar cholangiocarcinoma: A single-institution experience in China. WJG 2016;22(8):2601. [doi: 10.3748/wjg.v22.i8.2601]

32. Pawlik TM, Pulitano C, Alexandrescu S, Gamblin TC, Ferrone C, Sotiropoulos G, et al. Intrahepatic cholangiocarcinoma: An international, multi-institutional analysis of prognostic factors and lymph node assessment. JCO 2011 Feb 01;29(4_suppl):162-162. [doi: 10.1200/jco.2011.29.4_suppl.162] 
33. Amini N, Ejaz A, Spolverato G, Maithel SK, Kim Y, Pawlik TM. Management of Lymph Nodes During Resection of Hepatocellular Carcinoma and Intrahepatic Cholangiocarcinoma: A Systematic Review. J Gastrointest Surg 2014 Oct 10;18(12):2136-2148. [doi: 10.1007/s11605-014-2667-1]

34. Anderson C. Fluorodeoxyglucose PET imaging in the evaluation of gallbladder carcinoma and cholangiocarcinoma. Journal of Gastrointestinal Surgery 2004 Jan 01;8(1):90-97. [doi: 10.1016/j.gassur.2003.10.003]

35. Wakabayashi H, Akamoto S, Yachida S, Okano K, Izuishi K, Nishiyama Y, et al. Significance of fluorodeoxyglucose PET imaging in the diagnosis of malignancies in patients with biliary stricture. European Journal of Surgical Oncology (EJSO) 2005 Dec;31(10):1175-1179. [doi: 10.1016/j.ejso.2005.05.012]

36. Caudell JJ, Torres-Roca JF, Gillies RJ, Enderling H, Kim S, Rishi A, et al. The future of personalised radiotherapy for head and neck cancer. The Lancet Oncology 2017 May;18(5):e266-e273. [doi: 10.1016/s1470-2045(17)30252-8]

37. Lambin P, Leijenaar RT, Deist TM, Peerlings J, de Jong EE, van Timmeren J, et al. Radiomics: the bridge between medical imaging and personalized medicine. Nat Rev Clin Oncol 2017 Oct 4;14(12):749-762. [doi: 10.1038/nrclinonc.2017.141]

38. Limkin E, Sun R, Dercle L, Zacharaki E, Robert C, Reuzé S, et al. Promises and challenges for the implementation of computational medical imaging (radiomics) in oncology. Annals of Oncology 2017 Jun;28(6):1191-1206. [doi: 10.1093/annonc/mdx034]

39. Verma V, Simone C, Krishnan S, Lin S, Yang J, Hahn S. The rise of radiomics and implications for oncologic management. J Natl Cancer Inst 2017;109(7). [doi: 10.1093/jnci/djx055]

40. Peng Y, Zhou C, Lin P, Wen D, Wang X, Zhong X, et al. Preoperative Ultrasound Radiomics Signatures for Noninvasive Evaluation of Biological Characteristics of Intrahepatic Cholangiocarcinoma. Academic Radiology 2020 Jun;27(6):785-797. [doi: 10.1016/j.acra.2019.07.029]

41. Ji G, Zhu F, Zhang Y, Liu X, Wu F, Wang K, et al. A radiomics approach to predict lymph node metastasis and clinical outcome of intrahepatic cholangiocarcinoma. Eur Radiol 2019 Mar 26;29(7):3725-3735. [doi: 10.1007/s00330-019-06142-7]

42. Zhao L, Ma X, Liang M, Li D, Ma P, Wang S, et al. Prediction for early recurrence of intrahepatic mass-forming cholangiocarcinoma: quantitative magnetic resonance imaging combined with prognostic immunohistochemical markers. Cancer Imaging 2019 Jul 15;19(1). [doi: 10.1186/s40644-019-0234-4]

43. Liang W, Xu L, Yang P, Zhang L, Wan D, Huang Q, et al. Novel Nomogram for Preoperative Prediction of Early Recurrence in Intrahepatic Cholangiocarcinoma. Front. Oncol 2018 Sep 4;8. [doi: 10.3389/fonc.2018.00360]

44. Romagnuolo J, Bardou M, Rahme E, Joseph L, Reinhold C, Barkun AN. Magnetic Resonance Cholangiopancreatography. Ann Intern Med 2003 Oct 07;139(7):547. [doi: 10.7326/0003-4819-139-7-200310070-00006]

45. Xie J, Wen D, Liang L, Jia Y, Gao L, Lei J. Evaluating the Validity of Current Mainstream Wearable Devices in Fitness Tracking Under Various Physical Activities: Comparative Study. JMIR Mhealth Uhealth 2018 Apr 12;6(4):e94 [FREE Full text] [doi: 10.2196/mhealth.9754] [Medline: 29650506]

46. Wen D, Zhang X, Liu X, Lei J. Evaluating the Consistency of Current Mainstream Wearable Devices in Health Monitoring: A Comparison Under Free-Living Conditions. J Med Internet Res 2017 Mar 07;19(3):e68 [FREE Full text] [doi: 10.2196/jmir.6874] [Medline: 28270382]

\section{Abbreviations}

ADC: apparent diffusion coefficient

AUC: area under the curve

DD: differentiation degree

DDR: data dimensionality reduction

DICOM: Digital Imaging and Communications in Medicine

DWI: diffusion-weighted imaging

ECC: extrahepatic cholangiocarcinoma

ICC: intraclass correlation coefficient

LNM: lymph node metastasis

MRI: magnetic resonance imaging

NPV: negative predictive value

PACS: picture archiving and communication system

PPV: positive predictive value

PSO-SVM: particle swarm optimization and support vector machine

ROC: receiver operating characteristic

ROI: region of interest

T1WI: T1-weighted imaging

T2WI: T2-weighted imaging

18F-FDG-PET/CT: 18-fluorodeoxyglucose positron emission tomography/computerized tomography 
Edited by C Lovis, G Eysenbach; submitted 17.08.20; peer-reviewed by F Wang, D Liu; comments to author 06.09.20; accepted 18.09.20; published 05.10.20

Please cite as:

Yao X, Huang X, Yang C, Hu A, Zhou G, Ju M, Lei J, Shu J

A Novel Approach to Assessing Differentiation Degree and Lymph Node Metastasis of Extrahepatic Cholangiocarcinoma: Prediction Using a Radiomics-Based Particle Swarm Optimization and Support Vector Machine Model

JMIR Med Inform 2020;8(10):e23578

URL: https://medinform.jmir.org/2020/10/e23578

doi: $\underline{10.2196 / 23578}$

PMID: 33016889

(CXiaopeng Yao, Xinqiao Huang, Chunmei Yang, Anbin Hu, Guangjin Zhou, Mei Ju, Jianbo Lei, Jian Shu. Originally published in JMIR Medical Informatics (http://medinform.jmir.org), 05.10.2020. This is an open-access article distributed under the terms of the Creative Commons Attribution License (https://creativecommons.org/licenses/by/4.0/), which permits unrestricted use, distribution, and reproduction in any medium, provided the original work, first published in JMIR Medical Informatics, is properly cited. The complete bibliographic information, a link to the original publication on http://medinform.jmir.org/, as well as this copyright and license information must be included. 